\title{
Nutritional issues in patients with cancer
}

\author{
Duk Hwan Kim \\ Digestive Disease Center, CHA Bundang Medical Center, CHA University, Seongnam, Korea
}

Cancer is a catabolic inflammatory disease that causes patients to often experience weight loss, or even cachexia in severe cases. Undernourishment in patients with cancer impairs the quality of life and therapeutic response, further leading to poor prognosis. Active and frequent nutritional screening and assessment using valid tools are important for fast and appropriate nutritional intervention. Additionally, a suitable individualized nutritional intervention strategy should be established based on the nutritional assessment result. In general, nutritional intervention begins with nutritional counseling of patients diagnosed with cancer, and a well-planned nutritional counseling improves the treatment adherence and nutritional status. When planning nutritional supplementation for cancer patients, specific nutrients, including amino acids and fatty acids, should be considered. However, there has been no consistent result showing that any particular nutrient significantly improves the prognosis of cancer patients. Hence, continuous attention from clinical physicians is needed to plan nutritional improvement in patients with cancer. (Intest Res 2019;17:455-462)

Key Words: Neoplasms; Nutrition assessment; Nutrition therapy

\section{INTRODUCTION}

Nutritional problems are often encountered during the treatment of cancer. A prospective observational study reported that $51.1 \%$ of all cancer patients presented nutritional impairment, and $64 \%$ of patients showed reduction in weight 6 months after diagnosis. ${ }^{1}$ Weight loss, especially cachexia, are widely recognized as not only reduced physical function and quality of life, but also poor prognostic factors in cancer patients. Classically, BMI is often used for measuring nutritional status of a patient, and recent studies have focused more on sarcopenia. ${ }^{2}$ However, nutritional problems are complex and vary depending on the location and stage of cancer. ${ }^{1,3}$ Therefore, nutritional support for cancer patients should be based on the assessment of each patient's condition and appropriate planning of the outcome. In this review, we will look at what should be consid-

Received June 10, 2019. Revised July 3, 2019. Accepted July 5, 2019. Correspondence to Duk Hwan Kim, Digestive Disease Center, CHA Bundang Medical Center, CHA University, 59 Yatap-ro, Bundang-gu, Seongnam 13496 , Korea. Tel: +82-31-780-1925, Fax:+82-31-780-1876, E-mail: teires.d.kim@ gmail.com ered to prevent malnutrition by providing adequate nutrition to cancer patients.

\section{ALTERATION OF ENERGY METABOLISM IN CANCER PATIENTS}

Cachexia, commonly seen in cancer patients, is not simply caused by malnutrition due to anorexia. It is a more complex condition including reduced intake, metabolic dysfunction, and increased energy requirement. This process involves a variety of inflammatory cytokines in cancer cells, alterations in protein and lipid metabolism, and an imbalance in the production and degradation processes of muscle proteins.

Dysfunction in the regulation of human inflammatory process is observed in several diseases, including cancer. Researches have shown that increased inflammatory cytokines, such as TNF- $\alpha$ and interleukin-6 (IL-6), play critical roles in the nutritional metabolism of cancer patients. ${ }^{4}$ TNF- $\alpha$ is a cytokine related to cachexia, and it was originally called cachectin. ${ }^{5}$ It has long been known that TNF- $\alpha$ infusion causes loss of skeletal muscle mass in mice, and TNF- $\alpha$ blocking immunoglobulin 
reduced muscle loss in tumor-bearing rats. ${ }^{6,7}$ Although it is controversial if TNF- $\alpha$ levels in blood are higher in cancer patients, it is believed that TNF- $\alpha$ activates nuclear factor kappalight-chain-enhancer of activated B cells (NF- $\mathrm{KB}$ ) and promotes the degradation of proteins through a pathway independent of the ubiquitin-proteasome pathway. ${ }^{8}$ Furthermore, TNF- $\alpha$ activates other cytokines and can cause symptoms associated with cachexia, such as anorexia. ${ }^{9}$ A study using an antiTNF- $\alpha$ agent, etanercept, in cancer patients reported that etanercept improved chemotherapy adherence and fatigue during cancer treatment. ${ }^{10}$ IL-6 is also believed to play a very important role in cancer-related cachexia. In a study using ApcMin/+ mice, elevation of IL-6 did not induce cachexia in the tumor-free mice. However, elevation of IL-6 was associated with decreased skeletal muscle and fat mass, and increased tumor burden in ApcMin/+ mice. ${ }^{11}$ In cancer patients, IL-6 increases the acute phase reactants such as CRP through signal transducer and activator of transcription 3 (STAT3), and it is associated with muscle wasting. ${ }^{12}$ However, in a study of lung cancer patients, administration of humanized anti-IL-6 antibody was effective in alleviating symptoms such as anorexia, but it did not induce weight gain. ${ }^{13}$ Therefore, it is observed that cancer-associated cachexia is not simply related to one cytokine, but is influenced by the interactions of various signaling substances.

In catabolic diseases, various hormones and cytokines regulate protein production and degradation through the ubiquitin-proteasome pathway, autophagy, and transforming growth factor beta family ligands. Up-regulation of the ubiquitin-proteasome pathway by catabolic stress in several animal tumor models has been associated with muscle wasting. Myofibrillar components of muscle protein are mainly disintegrated in the ubiquitin-proteasome pathway, and this leads to decreased muscle strength. ${ }^{14}$ Moreover, the stress hormones and inflammatory cytokines promote autophagy and mitochondrial dysfunction, which lead to muscle atrophy. These processes are regulated by atrogenes and transcription factors, such as NF$\kappa \mathrm{B}$ and forkhead box protein $\mathrm{O}(\mathrm{FOXO}) .{ }^{14}$ Especially in cancer patients, chemotherapy itself and malabsorption by complications of chemotherapy, such as mucositis, can directly induce muscle wasting. ${ }^{15}$

It is well known that impairment of carbohydrate metabolism occurs in cancer patients. Cancer cells show high glycolysis, and glucose is produced by gluconeogenesis in the liver using lactate produced by the cancer cells. ${ }^{16}$ During cancer-associated cachexia, increased level of insulin-like growth factor-1 is observed, resulting in insulin resistance. ${ }^{17}$ Thus, most of the glucose induced is used by the cancer cells; hence, cancer patients have a very high energy demand. However, in actual clinical studies, insulin resistance is not inevitably associated with weight loss. ${ }^{18}$

Lipid metabolism is also impaired in cancer patients. The loss of adipose tissue by metabolic impairment further promotes cancer-associated cachexia. The accumulation of triglycerides in the cytoplasm of adipocytes is the most efficient method of energy storage. Free fatty acid and glycerol from triglyceride increase in patients with cancer-associated cachexia. ${ }^{19}$ Higher lipolysis and fat oxidation rates were observed in cancer patients losing weight as compared to that of healthy control. ${ }^{20}$ This lipolysis was promoted by hormones, pro-inflammatory cytokines, and lipid-mobilizing factor. ${ }^{21}$ One interesting finding of lipid metabolism in cancer patients is fat browning. White adipose tissue cells convert to brown adipose tissue-like cells (beige cells) during cancer-associated cachexia, similar to the mechanism in cold environments. ${ }^{22}$ The abundant mitochondrial components of beige cells produce heat (thermogenesis) from ATP synthesis through increased uncoupling protein- $-1 .{ }^{17}$ Fat browning in cancer-associated cachexia represents imbalance of homeostasis and contributes to catabolic wasting unlike normal physiologic conditions.

\section{IMPACT OF MALNUTRITION ON TREATMENT OUTCOMES IN CANCER PATIENTS}

Although the stage and type of cancer and the response to chemotherapy are the most important prognostic factors, many studies have reported that patients who maintained weight gain presented better prognosis than those who did not. Malnutrition is associated with longer hospital stay and higher rate of admission, delayed wound healing, deterioration of the immune system, and cancer-associated death. ${ }^{23-25}$ An important association beyond just cause and effect has been elucidated between malnutrition and aggravation of the disease. In a multicenter study investigating the prevalence of malnutrition in patients during cancer treatment, old age, hospital stay, and metastasis showed an association with malnutrition. Moreover, malnutrition was associated with higher infection rate and longer hospital stay; ${ }^{26}$ therefore, mere focus on increasing the weight of cancer patients does not affect the patient's clinical prognosis positively.

Sarcopenia in patients with cachexia has recently been noted for contributing to poor prognosis in cancer patients. In a study that investigated prognosis of patients with cholangio- 
carcinoma after major surgery, preoperative sarcopenia was associated with longer hospital stay, higher rate of liver failure, and higher postoperative infection rate. ${ }^{27}$ Sarcopenia showed positive association with increased toxicity from chemotherapy and faster tumor progression in patients with metastatic breast cancer, ${ }^{28}$ and was related to lower survival rate. In a retrospective study analyzing 229 stage III colon cancer patients, one standard deviation decrement of the psoas muscle mass index was related to an increased hazard of overall mortality in multivariate analysis. ${ }^{29}$ Likewise, in a prospective study of patients with foregut cancer treated with neoadjuvant chemotherapy, loss of skeletal muscle mass was a predictor of reduced survival rate. ${ }^{30}$ Several other studies of different cancer types reported that sarcopenia adversely affected the prognosis associated with cancer treatment and was associated with complications.

The quality of social, psychological, and physical life can be greatly reduced because of the cancer pathology itself. The nutritional status of a cancer patient is influenced by the quality of life, because weight loss and decreased appetite are significantly related to the quality of life. ${ }^{31,32}$ However, the poor quality of life in cancer patients is not solved simply by improving the nutritional status. A prospective randomized control trial showed that dietary intervention involving regular nutritional counseling in patients with solid tumor during chemotherapy was not significantly related to a better quality of life as compared to the control. The results were consistent even if the patients had better nutritional status through nutritional counseling. The quality of life considerably improved in patients with significant tumor regression. ${ }^{33}$ These results show that the relationship between cancer, malnutrition, and quality of life is complex.

\section{NUTRITIONAL SCREENING AND ASSESSMENT IN CANCER PATIENTS}

Assessing weight loss in cancer patients has been used since long as the simplest and most effective way to determine malnutrition. Although BMI can be disturbed by ascites and body edema, many studies showed that BMI and unintended weight loss were the methods used to monitor malnutrition in patients and predict poor prognosis. Even though various nutritional assessment tools have been introduced and actively used, BMI still plays an important role. A study investigating 3,779 patients with colorectal cancer, between 1972 and 2017, revealed that the underweight group showed a significantly worse overall survival rate as compared to the normal weight group in stage III and IV cancer patients. More than $10 \%$ of weight reduction from pre-diagnosis to post-diagnosis showed a significant relation with poorer overall survival. ${ }^{34}$ Moreover, in a prospective study analyzing 82 metastatic breast cancer patients from 2011 to 2012, overweight patients showed a significant association with lower rate of cancer mortality as compared to the normal weight patients. ${ }^{35}$ Excessive nutrition is not recommended when considering the overall disease mortality including metabolic diseases. Furthermore, the prognosis is worse in overweight patients when there is accompanied muscle loss. Thus, it is obvious that simply maintaining the weight could play a protective role against cancer mortality.

Traditionally, serum concentrations of liver proteins, such as albumin, have been associated with the nutritional status of patients. Therefore, a lack of these proteins indicated undernourishment and was used as an indicator of active nutritional support. ${ }^{36}$ However, since albumin has a long half-life and is inhibited by inflammatory cytokines including TNF- $\alpha$ and IL6 , which are elevated in cancer patients, it cannot be an immediate and accurate indicator of malnutrition. ${ }^{37}$

Nowadays, instead of using only 1 specific parameter to evaluate the patient, several factors are combined to assess the nutritional risk and evaluate the nutritional status. The Malnutrition Universal Screening Tool, Nutrition Risk Screening 2002, Mini Nutritional Assessment Short Form, and Malnutrition Screening Tool are validated and widely-used nutritional risk screening tools. ${ }^{38}$ The purpose of nutritional risk screening is to emphasize the importance of nutritional support and promote early intervention to ultimately prevent poor outcomes due to malnutrition. Therefore, these screening tests should be easy to apply and interpret. Although they have different target patients, all the screening tools use parameters of BMI, weight change, accompanying disease, and degree of food intake. ${ }^{39}$ The strategy for screening nutritional risk in cancer patients should adopt an individualized strategy for each medical institution considering the applicability of each tool. Although there is a lack of clear evidence eliciting the clinical benefits of nutritional screening tools in cancer patients, no studies have proved the inefficacy of nutritional risk screening tools. ${ }^{40-42}$ In fact, there are certain benefits of screening for nutritional risk depending on the type and treatment of cancers. Therefore, the European Society for Clinical Nutrition and Metabolism (ESPEN) guideline recommends that there should be regular evaluation of nutritional intake, weight change, and BMI, that starts during cancer diagnosis and is repeated depending on the stability of 
Table 1. Nutritional Screening and Assessment Tools

\begin{tabular}{|c|c|}
\hline Tool & Evaluation item \\
\hline \multicolumn{2}{|l|}{ Screening tool } \\
\hline Malnutrition Universal Screening Tool & BMI $\left(\mathrm{kg} / \mathrm{m}^{2}\right)$, weight loss $(\%)$, nutritional intake \\
\hline \multirow[t]{2}{*}{ Nutrition Risk Screening 2002} & $\begin{array}{l}\text { Initial screening: BMI }\left(\mathrm{kg} / \mathrm{m}^{2}\right) \text {, weight loss (during } 3 \text { months), nutritional intake (during a week), disease } \\
\text { severity }\end{array}$ \\
\hline & Final screening: impaired nutritional status (score), disease severity (score), age (additional score) \\
\hline Mini Nutritional Assessment Short Form & $\begin{array}{l}\text { Food intake, weight loss, mobility, psychological stress, neuropsychological problems, BMI }\left(\mathrm{kg} / \mathrm{m}^{2}\right) \text {, calf } \\
\text { circumference }(\mathrm{cm})\end{array}$ \\
\hline Malnutrition Screening Tool & Weight loss, appetite \\
\hline \multicolumn{2}{|l|}{ Assessment tool } \\
\hline \multirow{2}{*}{$\begin{array}{l}\text { Patient-generated-subjective global } \\
\text { assessment }\end{array}$} & Patient: weight, symptom, nutritional intake, activity \\
\hline & Medical staff: diagnosis, nutritional requirement, physical examination \\
\hline Nutrition Risk Index & $(1.519 \times$ serum albumin, $\mathrm{g} / \mathrm{dL})+(41.7 \times$ present weight $[\mathrm{kg}] /$ ideal body weight $[\mathrm{kg}])$ \\
\hline
\end{tabular}

the clinical situation. ${ }^{39}$

If the patient is predisposed to have a nutritional risk, an assessment of the patient's nutritional status must be performed. Comprehensive nutritional assessment provides an objective goal for individualized nutritional care of the patients, ultimately improving the clinical outcomes by protecting the patients from treatment complications, and improving both the treatment outcomes and quality of life. Therefore, the nutritional assessment of cancer patients should be repeated periodically, and not just once. There are many nutritional assessment tools used in researches and clinical practice. Although each nutritional assessment tool varies slightly, most tools consist of the patient's medical history, food intake, physical activity level, weight change, other anthropometric measures, and laboratory test results. ${ }^{43}$ Among the various tools available, the patient-generated-subjective global assessment (PG-SGA) has been developed specifically for cancer patients. PG-SAG is a tool that evaluates the clinical aspects, including physical examination and accompanying diseases, and confirms the change in the patient's weight or dietary intake, presence or absence of gastrointestinal symptoms that may affect the dietary intake, and expresses a numerical score. ${ }^{44}$ The start and posttreatment follow-up of nutritional intervention are based on this score. PG-SGA is a valid and dependable tool that provides a reference to identify and classify the nutritional state of a cancer patient. Nutrition Risk Index is another commonly used assessment tools. ${ }^{45,46}$ Reduced muscle mass can be measured objectively using dual X-ray absorptiometry, CT scans, or bioimpedance analysis (Table 1).

\section{NUTRITIONAL INTERVENTIONS}

When a patient is diagnosed with cancer for the first time, the doctor should pay special attention to nutrition to prevent the patient from following detrimental diets. Doctors, as scientists, are naturally alert towards non-evidence-based treatments and excessive dietary imbalances, such as fad diets, that have a negative impact on the financial state as well as on the clinical prognosis. A fad diet is an alternative medicine comprising of extremely high or low intake of certain foods, which will most likely result in nutritional imbalance. Furthermore, there is no strong evidence supporting the beneficial effects of the fad diets on cancer treatment. ${ }^{39}$ Cancer patients are mentally vulnerable and tend to be obsessed with food control, and thus the patient could actively intervene in the cancer treatment process. Hence, doctors treating cancer should understand the characteristics of these patients and implement appropriate interventions. Nutritional counseling and diet advice are therefore very important in cancer patients at nutritional risk during diagnosis, and these can be conducted from 3 perspectives. First, the amount of food consumed should be increased. Second, the composition of the food should be balanced along with high-energy intake from a nutritional point of view. Third, nutritional supplements that can be ingested orally should be considered. ${ }^{42}$ Cochrane review reported that nutritional counseling had a beneficial effect on improving the weight reduction and quality of life in patients with cancer by analyzing randomized controlled trials. ${ }^{42}$ In addition to nutritional counseling, there have been studies wherein patients who consumed oral nutritional supplements demonstrated 
better weight gain than those who did not. A systematic review and meta-analysis of 13 studies reported that oral nutritional interventions had a beneficial effect on the quality of life. ${ }^{47}$ On the other hand, another meta-analysis showed that oral nutritional supplementation improved the quality of life of patients but did not reduce the mortality in cancer patients. ${ }^{47}$

From the perspective of overcoming sarcopenia, high-protein diets can be considered. Patients with acute or chronic diseases need $1.5 \mathrm{~g} / \mathrm{kg}$ of protein per day, but one study found that $35 \%$ of cancer patients consumed fewer proteins than required. ${ }^{48,49}$ A randomized, controlled, double-blind, parallelgroup designed study using experimental high-protein diet in cancer patients revealed that the high-protein diet group showed significantly better muscle synthesis than the control group. ${ }^{50}$ Although leucine is the most important amino acid in muscle synthesis, there are no consistent results showing that high doses of leucine will help prognosis in patients with cancer. Moreover, a recent experimental study showed that high level of leucine could induce tamoxifen resistance in estrogen-receptor positive breast cancer patients. ${ }^{51}$ Therefore, when using high dose of a specific nutrient, a thoughtful evaluation is necessary. Glutamine is also considered a nutritional supplement for cancer patients. Glutamine is a nonessential amino acid that serves an important role in the metabolism and immune system. Glutamine levels are reduced in cancer patients because glutamine is used as the energy source by cancer cells. Glutamine supplementation is usually focused on preventing side effects that may occur during chemotherapy or radiotherapy, especially in gastrointestinal mucosal protection. A prospective study investigating the beneficial effect of a parenteral glutamine supplementation in 24 metastatic colorectal cancer patients during chemotherapy revealed that glutamine supplementation was associated with a significant reduction in mucositis and ulceration of the upper gastrointestinal mucosa. ${ }^{52}$ However, additional prospective studies are needed to determine the relationship between prognosis and glutamine supplementation in cancer patients and the effect of oral and intravenous glutamine.

Omega-3 fatty acids can also be expected to provide nutritional supplementation in cancer patients. Unlike omega-6 fatty acids, omega-3 fatty acids have anti-inflammatory effects and do not promote angiogenesis. ${ }^{53}$ Therefore, supplementing omega-3 in cancer patients has the advantage of providing nutrition, especially fat, without stimulating tumor growth. In some studies, ${ }^{54,55}$ omega-3 supplementation was shown to help weight gain in cancer patients, while other systematic re- view studies showed that there was unclear evidence of the beneficial effect of omega-3 in improving cachexia in cancer patients. ${ }^{56,57}$ However, since the intake of omega-3 fatty acids rarely causes serious adverse effects, the ESPEN guideline recommended supplementing omega-3 fatty acids in cancer patients having weight loss. ${ }^{39}$

\section{CONCLUSION}

There is a lack of attention towards the nutritional status of cancer patients in clinical practice. However, monitoring and intervention of nutritional status not only affect the quality of life of the patient, but also the treatment response and clinical outcomes. Since no strategy particularly good at improving the nutritional status in cancer patients has yet been developed, it is important that individualized treatments be performed for each patient according to their nutritional assessment. Moreover, continuous vigilance regarding factors affecting nutritional status in patients with cancer is necessary.

\section{FINANCIAL SUPPORT}

The author received no financial support for the research, authorship, and/or publication of this article.

\section{CONFLICT OF INTEREST}

No potential conflict of interest relevant to this article was reported.

\section{AUTHOR CONTRIBUTION}

Writing and approval of final manuscript: Kim DH.

\section{ORCID}

Kim DH

https://orcid.org/0000-0003-3841-5802

\section{REFERENCES}

1. Muscaritoli M, Lucia S, Farcomeni A, et al. Prevalence of malnutrition in patients at first medical oncology visit: the PreMiO study. Oncotarget 2017;8:79884-79896.

2. Davis MP, Panikkar R. Sarcopenia associated with chemotherapy and targeted agents for cancer therapy. Ann Palliat Med 2019;8:86-101. 
3. Hébuterne X, Lemarié E, Michallet M, de Montreuil CB, Schneider SM, Goldwasser F. Prevalence of malnutrition and current use of nutrition support in patients with cancer. JPEN J Parenter Enteral Nutr 2014;38:196-204.

4. Laine A, Iyengar P, Pandita TK. The role of inflammatory pathways in cancer-associated cachexia and radiation resistance. Mol Cancer Res 2013;11:967-972.

5. Beutler B, Greenwald D, Hulmes JD, et al. Identity of tumour necrosis factor and the macrophage-secreted factor cachectin. Nature 1985;316:552-554.

6. Costelli P, Carbó N, Tessitore L, et al. Tumor necrosis factor-alpha mediates changes in tissue protein turnover in a rat cancer cachexia model. J Clin Invest 1993;92:2783-2789.

7. Fong Y, Moldawer LL, Marano M, et al. Cachectin/TNF or IL-1 alpha induces cachexia with redistribution of body proteins. Am J Physiol 1989;256(3 Pt 2):R659-R665.

8. Han Y, Weinman S, Boldogh I, Walker RK, Brasier AR. Tumor necrosis factor-alpha-inducible IkappaBalpha proteolysis mediated by cytosolic m-calpain: a mechanism parallel to the ubiquitin-proteasome pathway for nuclear factor-kappab activation. J Biol Chem 1999;274:787-794.

9. Argilés JM, Busquets S, López-Soriano FJ. Anti-inflammatory therapies in cancer cachexia. Eur J Pharmacol 2011;668 Suppl 1:S81-S86.

10. Monk JP, Phillips G, Waite R, et al. Assessment of tumor necrosis factor alpha blockade as an intervention to improve tolerability of dose-intensive chemotherapy in cancer patients. J Clin Oncol 2006;24:1852-1859.

11. Baltgalvis KA, Berger FG, Pena MM, Davis JM, Muga SJ, Carson JA. Interleukin-6 and cachexia in ApcMin/+ mice. Am J Physiol Regul Integr Comp Physiol 2008;294:R393-R401.

12. Bonetto A, Aydogdu T, Kunzevitzky N, et al. STAT3 activation in skeletal muscle links muscle wasting and the acute phase response in cancer cachexia. PLoS One 2011;6:e22538.

13. Bayliss TJ, Smith JT, Schuster M, Dragnev KH, Rigas JR. A humanized anti-IL-6 antibody (ALD518) in non-small cell lung cancer. Expert Opin Biol Ther 2011;11:1663-1668.

14. Cohen S, Nathan JA, Goldberg AL. Muscle wasting in disease: molecular mechanisms and promising therapies. Nat Rev Drug Discov 2015;14:58-74.

15. Kodera Y. More than 6 months of postoperative adjuvant chemotherapy results in loss of skeletal muscle: a challenge to the current standard of care. Gastric Cancer 2015;18:203-204.

16. Holroyde CP, Skutches CL, Boden G, Reichard GA. Glucose metabolism in cachectic patients with colorectal cancer. Cancer Res 1984;44(12 Pt 1):5910-5913.
17. Porporato PE. Understanding cachexia as a cancer metabolism syndrome. Oncogenesis 2016;5:e200.

18. Agustsson T, D'souza MA, Nowak G, Isaksson B. Mechanisms for skeletal muscle insulin resistance in patients with pancreatic ductal adenocarcinoma. Nutrition 2011;27:796-801.

19. Das SK, Hoefler G. The role of triglyceride lipases in cancer associated cachexia. Trends Mol Med 2013;19:292-301.

20. Zuijdgeest-van Leeuwen SD, van den Berg JW, Wattimena JL, et al. Lipolysis and lipid oxidation in weight-losing cancer patients and healthy subjects. Metabolism 2000;49:931-936.

21. Tsoli M, Swarbrick MM, Robertson GR. Lipolytic and thermogenic depletion of adipose tissue in cancer cachexia. Semin Cell Dev Biol 2016;54:68-81.

22. Petruzzelli M, Wagner EF. Mechanisms of metabolic dysfunction in cancer-associated cachexia. Genes Dev 2016;30:489501.

23. Correia MI, Waitzberg DL. The impact of malnutrition on morbidity, mortality, length of hospital stay and costs evaluated through a multivariate model analysis. Clin Nutr 2003;22:235239.

24. Pressoir M, Desné S, Berchery D, et al. Prevalence, risk factors and clinical implications of malnutrition in French Comprehensive Cancer Centres. Br J Cancer 2010;102:966-971.

25. Virizuela JA, Camblor-Álvarez M, Luengo-Pérez LM, et al. Nutritional support and parenteral nutrition in cancer patients: an expert consensus report. Clin Transl Oncol 2018;20:619629.

26. Marshall KM, Loeliger J, Nolte L, Kelaart A, Kiss NK. Prevalence of malnutrition and impact on clinical outcomes in cancer services: a comparison of two time points. Clin Nutr 2019;38: 644-651.

27. Otsuji H, Yokoyama Y, Ebata T, et al. Preoperative sarcopenia negatively impacts postoperative outcomes following major hepatectomy with extrahepatic bile duct resection. World J Surg 2015;39:1494-1500.

28. Prado CM, Baracos VE, McCargar LJ, et al. Sarcopenia as a determinant of chemotherapy toxicity and time to tumor progression in metastatic breast cancer patients receiving capecitabine treatment. Clin Cancer Res 2009;15:2920-2926.

29. Jung HW, Kim JW, Kim JY, et al. Effect of muscle mass on toxicity and survival in patients with colon cancer undergoing adjuvant chemotherapy. Support Care Cancer 2015;23:687694.

30. Daly LE, Ní Bhuachalla ÉB, Power DG, Cushen SJ, James K, Ryan AM. Loss of skeletal muscle during systemic chemotherapy is prognostic of poor survival in patients with foregut can- 
cer. J Cachexia Sarcopenia Muscle 2018;9:315-325.

31. O'Gorman P, McMillan DC, McArdle CS. Impact of weight loss, appetite, and the inflammatory response on quality of life in gastrointestinal cancer patients. Nutr Cancer 1998;32: 76-80.

32. Ollenschläger G, Thomas W, Konkol K, Diehl V, Roth E. Nutritional behavior and quality of life during oncological polychemotherapy: results of a prospective study on the efficacy of oral nutrition therapy in patients with acute leukaemia. Eur J Clin Invest 1992;22:546-553.

33. Ovesen L, Allingstrup L, Hannibal J, Mortensen EL, Hansen OP. Effect of dietary counseling on food intake, body weight, response rate, survival, and quality of life in cancer patients undergoing chemotherapy: a prospective, randomized study. J Clin Oncol 1993;11:2043-2049.

34. Shahjehan F, Merchea A, Cochuyt JJ, Li Z, Colibaseanu DT, Kasi PM. Body mass index and long-term outcomes in patients with colorectal cancer. Front Oncol 2018;8:620.

35. Alarfi H, Salamoon M, Kadri M, et al. The impact of baseline body mass index on clinical outcomes in metastatic breast cancer: a prospective study. BMC Res Notes 2017;10:550.

36. Nazha B, Moussaly E, Zaarour M, Weerasinghe C, Azab B. Hypoalbuminemia in colorectal cancer prognosis: nutritional marker or inflammatory surrogate? World J Gastrointest Surg 2015;7:370-377.

37. Fuhrman MP, Charney P, Mueller CM. Hepatic proteins and nutrition assessment. J Am Diet Assoc 2004;104:1258-1264.

38. Isenring E, Elia M. Which screening method is appropriate for older cancer patients at risk for malnutrition? Nutrition 2015; 31:594-597.

39. Arends J, Bachmann P, Baracos V, et al. ESPEN guidelines on nutrition in cancer patients. Clin Nutr 2017;36:11-48.

40. Pan H, Cai S, Ji J, et al. The impact of nutritional status, nutritional risk, and nutritional treatment on clinical outcome of 2248 hospitalized cancer patients: a multi-center, prospective cohort study in Chinese teaching hospitals. Nutr Cancer 2013; 65:62-70.

41. Baldwin C, Spiro A, McGough C, et al. Simple nutritional intervention in patients with advanced cancers of the gastrointestinal tract, non-small cell lung cancers or mesothelioma and weight loss receiving chemotherapy: a randomised controlled trial. J Hum Nutr Diet 2011;24:431-440.

42. Baldwin C, Weekes CE. Dietary advice with or without oral nutritional supplements for disease-related malnutrition in adults. Cochrane Database Syst Rev 2011;(9):CD002008.

43. Huhmann MB, August DA. Review of American Society for
Parenteral and Enteral Nutrition (ASPEN) Clinical Guidelines for Nutrition Support in Cancer Patients: nutrition screening and assessment. Nutr Clin Pract 2008;23:182-188.

44. Bauer J, Capra S, Ferguson M. Use of the scored Patient-Generated Subjective Global Assessment (PG-SGA) as a nutrition assessment tool in patients with cancer. Eur J Clin Nutr 2002; 56:779-785.

45. Veterans Affairs Total Parenteral Nutrition Cooperative Study Group. Perioperative total parenteral nutrition in surgical patients. N Engl J Med 1991;325:525-532.

46. Guigoz Y, Vellas B, Garry PJ. Assessing the nutritional status of the elderly: the Mini Nutritional Assessment as part of the geriatric evaluation. Nutr Rev 1996;54(1 Pt 2):S59-S65.

47. Baldwin C, Spiro A, Ahern R, Emery PW. Oral nutritional interventions in malnourished patients with cancer: a systematic review and meta-analysis. J Natl Cancer Inst 2012;104: 371-385.

48. Deutz NE, Bauer JM, Barazzoni R, et al. Protein intake and exercise for optimal muscle function with aging: recommendations from the ESPEN Expert Group. Clin Nutr 2014;33:929936.

49. Prado CM, Lieffers JR, Bowthorpe L, Baracos VE, Mourtzakis M, McCargar LJ. Sarcopenia and physical function in overweight patients with advanced cancer. Can J Diet Pract Res 2013;74:69-74.

50. Deutz NE, Safar A, Schutzler S, et al. Muscle protein synthesis in cancer patients can be stimulated with a specially formulated medical food. Clin Nutr 201 1;30:759-768.

51. Saito Y, Li L, Coyaud E, et al. LLGL2 rescues nutrient stress by promoting leucine uptake in $\mathrm{ER}(+)$ breast cancer. Nature 2019; 569:275-279.

52. Decker-Baumann C, Buhl K, Frohmüller S, von Herbay A, Dueck M, Schlag PM. Reduction of chemotherapy-induced side-effects by parenteral glutamine supplementation in patients with metastatic colorectal cancer. Eur J Cancer 1999;35: 202-207.

53. Caughey GE, Mantzioris E, Gibson RA, Cleland LG, James MJ. The effect on human tumor necrosis factor alpha and interleukin 1 beta production of diets enriched in n-3 fatty acids from vegetable oil or fish oil. Am J Clin Nutr 1996;63:116-122.

54. van der Meij BS, van Bokhorst-de van der Schueren MA, Langius JA, Brouwer IA, van Leeuwen PA. N-3 PUFAs in cancer, surgery, and critical care: a systematic review on clinical effects, incorporation, and washout of oral or enteral compared with parenteral supplementation. Am J Clin Nutr 2011;94:12481265 
55. Colomer R, Moreno-Nogueira JM, García-Luna PP, et al. N-3 fatty acids, cancer and cachexia: a systematic review of the literature. Br J Nutr 2007;97:823-831.

56. Ries A, Trottenberg P, Elsner F, et al. A systematic review on the role of fish oil for the treatment of cachexia in advanced cancer: an EPCRC cachexia guidelines project. Palliat Med
2012;26:294-304.

57. Mazzotta P, Jeney CM. Anorexia-cachexia syndrome: a systematic review of the role of dietary polyunsaturated fatty acids in the management of symptoms, survival, and quality of life. J Pain Symptom Manage 2009;37:1069-1077. 